\title{
An Eigenvalue-Eigenvector Method for Solving a System of Fractional Differential Equations with Uncertainty
}

\author{
M. R. Balooch Shahriyar, ${ }^{1}$ F. Ismail, ${ }^{2}$ S. Aghabeigi, ${ }^{3}$ A. Ahmadian, ${ }^{2,4}$ and S. Salahshour ${ }^{5}$ \\ ${ }^{1}$ Department of Mathematics, Kerman Branch, Islamic Azad University, P.O. Box 9189945, Kerman, Iran \\ ${ }^{2}$ Institute for Mathematical Research, Universiti Putra Malaysia, 43400 Serdang, Selangor, Malaysia \\ ${ }^{3}$ Department of Mathematics, Mobarakeh Branch, Islamic Azad University, P.O. Box 9176754, Mobarakeh, Iran \\ ${ }^{4}$ Department of Mathematics, Science Faculty, Universiti Putra Malaysia, 43400 Serdang, Selangor, Malaysia \\ ${ }^{5}$ Young Researchers and Elite Club, Mobarakeh Branch, Islamic Azad University, P.O. Box 9176754, Mobarakeh, Iran
}

Correspondence should be addressed to F. Ismail; fudziah@science.upm.edu.my

Received 13 March 2013; Revised 28 April 2013; Accepted 14 May 2013

Academic Editor: Tofigh Allahviranloo

Copyright (C) 2013 M. R. Balooch Shahriyar et al. This is an open access article distributed under the Creative Commons Attribution License, which permits unrestricted use, distribution, and reproduction in any medium, provided the original work is properly cited.

A new method is proposed for solving systems of fuzzy fractional differential equations (SFFDEs) with fuzzy initial conditions involving fuzzy Caputo differentiability. For this purpose, three cases are introduced based on the eigenvalue-eigenvector approach; then it is shown that the solution of system of fuzzy fractional differential equations is vector of fuzzy-valued functions. Then the method is validated by solving several examples.

\section{Introduction}

Recently, a lot of research has been focused on the application of fractional calculus, and such application is in the modelling of many physical and chemical processes as well as in engineering [1-5].

It has been found that the behavior of many physical systems can be properly described by using the fractional order system theory. Fractional derivatives provide an excellent instrument for the description of memory and hereditary properties of various materials and processes. The advantages or the real objects of the fractional order systems are that we have more degrees of freedom in the model and that a "memory" is included in the model. For example, the nonlinear oscillation of earthquake can be modeled with fractional derivatives [6]. In mechanics, fractional calculus plays an important role; for example, it has been successfully employed to model damping forces with memory effects to describe state feedback controllers $[7,8]$ and dynamics of interfaces between nanoparticles and substrates [9]. Due to its tremendous scope and applications in several disciplines, a considerable attention has been given to exact and numerical solutions of fractional differential equations [10-18]. The analytic results on the existence and uniqueness of solutions to the fractional differential equations have been studied by many authors $[19,20]$. From the numerical point of view, several methods have been presented to achieve the goal of highly accurate and reliable solutions for the fractional differential equations. The most commonly used methods are fractional differential transform method [21], operational matrix method [22, 23], finite difference method [24], and Haar wavelets method [25].

On the other hand, fuzzy differential equations have received considerable attention in dealing with various problems. So the development in this field has risen from the theoretical and practical perspectives [26-33].

Recently, Agarwal et al. [34] proposed the concept of solutions for fractional differential equations with uncertainty which was followed by the authors in $[35,36]$. They have considered Riemann-Liouville's differentiability to solve FFDEs which is a combination of the Hukuhara difference and the Riemann-Liouville derivative. In $[37,38]$, the authors considered the generalization of $\mathrm{H}$-differentiability for the fractional case. A lot of research has been devoted to find the accurate and efficient methods for solving fuzzy fractional dif ferential equations (FFDEs). It is well known that the exact 
solutions of most of the FFDEs cannot be found easily; therefore, in the recent years, attempts have been made to address this problem [39-41]. It is with this motivation that we introduce in this paper an eigenvalue-eigenvector method for solving fuzzy fractional differential equations (FFDEs).

Also regarding some defects of $\mathrm{H}$-differentiability [42], Bede et al. [43-46] discussed the limitations of the H-differentiability due to the shortcoming of the Hukuhara differentiability. So we motivated our interest to adopt our proposed method based on the generalized differentiability in the sense of fractional order which was introduced by $[37,38,41]$.

In this paper, we intend to investigate the solutions of systems of fractional differential equations with uncertainty which is called system of fuzzy fractional differential equations (SFFDEs). Here, we use the Riemann-Liouville derivative in the fuzzy concept, which applied the concept of the system of fractional derivatives under Caputo's differentiability by applying the Hukuhara difference, which is denoted as fuzzy Caputo's derivative. Similar to the deterministic cases, the construction of Caputo's derivatives is based on the definitions of the Riemann-Liouville derivatives in fuzzy cases.

This paper is organized as follows. In Section 2, we review the well-known definitions of fuzzy numbers, and some basic concepts are given. In Section 3, fuzzy Caputo's derivative is introduced, and the relation between Riemann-Liouville and Caputo's derivatives and some of its properties is considered. Consequently, the eigenvalue-eigenvector method for solving fuzzy fractional order linear systems with initial values under fuzzy Caputo's derivative is given, and three cases of eigenvalues are considered in Section 4. The proposed method is illustrated by solving several examples in Section 5 to depict the applicability and validity of the proposed method. Finally, conclusion is drawn in Section 6.

\section{Preliminaries}

The basic definition of fuzzy numbers is given in $[47,48]$.

We denote the set of all real numbers by $\mathbb{R}$, and the set of all fuzzy number on $\mathbb{R}$ is indicated by $\mathbb{E}$. A fuzzy number is a mapping $u: \mathbb{R} \rightarrow[0,1]$ with the following properties:

(a) $u$ is upper semicontinuous,

(b) $u$ is fuzzy convex; that is, $u(\lambda x+(1-\lambda) y) \geq$ $\min \{u(x), u(y)\}$ for all $x, y \in \mathbb{R}, \lambda \in[0,1]$,

(c) $u$ is normal; that is, $\exists x_{0} \in \mathbb{R}$ for which $u\left(x_{0}\right)=1$,

(d) supp $u=\{x \in \mathbb{R} \mid u(x)>0\}$ is the support of the $u$, and its closure $\operatorname{cl}(\operatorname{supp} u)$ is compact.

An equivalent parametric definition is also given in [4951] as follows.

Definition 1. A fuzzy number $u$ in parametric form is a pair $(\underline{u}, \bar{u})$ of functions $\underline{u}(r), \bar{u}(r), 0 \leq r \leq 1$, which satisfy the following requirements:

(1) $\underline{u}(r)$ is a bounded nondecreasing left continuous function in $(0,1]$ and right continuous at 0 ,

(2) $\bar{u}(r)$ is a bounded nonincreasing left continuous function in $(0,1]$ and right continuous at 0 ,
(3) $\underline{u}(r) \leq \bar{u}(r), 0 \leq r \leq 1$.

Moreover, we can also present the $r$-cut representation of fuzzy number as $[u]^{r}=[\underline{u}(r), \bar{u}(r)]$ for all $0 \leq r \leq 1$.

According to Zadeh's extension principle, operation of addition on $\mathbb{E}$ is defined by

$$
(u \oplus v)(x)=\sup _{y \in \mathbb{R}} \min \{u(y), v(x-y)\}, \quad x \in \mathbb{R},
$$

and scalar multiplication of a fuzzy number is given by

$$
(k \odot u)(x)= \begin{cases}u\left(\frac{x}{k}\right), & k>0, \\ \widetilde{0}, & k=0,\end{cases}
$$

where $\widetilde{0} \in \mathbb{E}$.

The Hausdorff distance between fuzzy numbers is given by $d: \mathbb{E} \times \mathbb{E} \rightarrow \mathbb{R}_{+} \bigcup\{0\}$,

$$
d(u, v)=\sup _{r \in[0,1]} \max \{|\underline{u}(r)-\underline{v}(r)|,|\bar{u}(r)-\bar{v}(\alpha)|\},
$$

where $u=(\underline{u}(r), \bar{u}(r))$ and $v=(\underline{v}(r), \bar{v}(r)) \subset \mathbb{R}$ is utilized in [43]. Then, it is easy to see that $d$ is a metric in $\mathbb{E}$ and has the following properties (see [33]):

(1) $d(u+w, v+w)=d(u, v)$, for all $u, v, w \in \mathbb{E}$,

(2) $d(k u, k v)=|k| d(u, v)$, for all $k \in \mathbb{R}, u, v \in \mathbb{E}$,

(3) $d(u+v, w+e) \leq d(u, w)+d(v, e)$, for all $u, v, w, e \in \mathbb{E}$,

(4) $(d, \mathbb{E})$ is a complete metric space.

Definition 2. Let $x, y \in \mathbb{E}$. If there exists $z \in \mathbb{E}$ such that $x=y+z$, then $z$ is called the H-difference of $x$ and $y$, and it is denoted by $x \ominus y$.

In this paper, the sign " $\ominus$ " always stands for $\mathrm{H}$-difference, and also note that $x \ominus y \neq x+(-1) y$.

\section{Fuzzy Caputo's Derivative}

In this section, the concept of fuzzy Caputo's derivatives is considered using the Hukuhara difference. We denote $C^{\mathbb{F}}[a, b]$ as a space of all fuzzy-valued functions which are continuous on $[a, b]$. Also, we denote the space of all Lebesgue integrable fuzzy-value functions on the bounded interval $[a, b] \subset \mathbb{R}$ by $L^{\mathbb{F}}[a, b]$. We denote the space of fuzzy-value functions $f(x)$ which have continuous $\mathrm{H}$-derivative up to order $n-1$ on $[a, b]$ such that $f^{(n-1)}(x) \in A C^{\mathbb{F}}([a, b])$ by $A C^{(n) \mathbb{F}}([a, b])$.

Now, we define the fuzzy Riemann-Liouville integral of fuzzy-valued function as follows.

Definition 3. Let $f(x) \in C^{\mathbb{F}}[a, b] \cap L^{\mathbb{F}}[a, b]$, the fuzzy Riemann-Liouville integral of fuzzy-valued function $f$ is defined as follows:

$$
\left(I_{a+}^{\beta} f\right)(x)=\frac{1}{\Gamma(\beta)} \int_{a}^{x} \frac{f(t) d t}{(x-t)^{1-\beta}}, \quad x>a, 0<\beta<1 .
$$


Since $f(x ; r)=[f(x ; r), \bar{f}(x ; r)]$, for all $0 \leq r \leq 1$, then we can indicate the fuzzy Riemann-Liouville integral of fuzzyvalued function $f$ based on the lower and upper functions as follows.

Theorem 4. Let $f(x) \in C^{\mathbb{F}}[a, b] \cap L^{\mathbb{F}}[a, b]$, the fuzzy Riemann-Liouville integral of fuzzy-valued function $f$ is defined as follows:

$$
\left(I_{a+}^{\beta} f\right)(x ; r)=\left[\left(I_{a+}^{\beta} \underline{f}\right)(x ; r),\left(I_{a+}^{\beta} \bar{f}\right)(x ; r)\right], \quad 0 \leq r \leq 1,
$$

where

$$
\begin{array}{ll}
\left(I_{a+}^{\beta} \underline{f}\right)(x ; r)=\frac{1}{\Gamma(\beta)} \int_{a}^{x} \frac{\bar{f}(t ; r) d t}{(x-t)^{1-\beta}}, \quad 0 \leq r \leq 1, \\
\left(I_{a+}^{\beta} \bar{f}\right)(x ; r)=\frac{1}{\Gamma(\beta)} \int_{a}^{x} \frac{\bar{f}(t ; r) d t}{(x-t)^{1-\beta}}, \quad 0 \leq r \leq 1 .
\end{array}
$$

Let $f \in C^{\mathbb{F}}((0, a]) \cap L^{\mathbb{F}}(0, a)$ be a given function such that $f(t, r)=[f(t ; r), \bar{f}(t ; r)]$ for all $t \in(0, a]$ and $0 \leq r \leq 1$. We define the fuzzy fractional Riemann-Liouville derivative of order $0<\beta<1$ of $f$,

$$
{ }^{\mathrm{RL}} D^{\beta} f(t)=\frac{1}{\Gamma(1-\beta)} \frac{d}{d t} \int_{0}^{t}(t-s)^{-\beta} f(s) d s,
$$

and in the parametric form,

$$
\begin{array}{r}
{ }^{\mathrm{RL}} D^{\beta} f(t, r) \\
=: \frac{1}{\Gamma(1-\beta)}\left[\frac{d}{d t} \int_{0}^{t}(t-s)^{-\beta} \underline{f}(s ; r) d s,\right. \\
\left.\frac{d}{d t} \int_{0}^{t}(t-s)^{-\beta} \bar{f}(s ; r) d s\right],
\end{array}
$$

provided that the equation defines a fuzzy number ${ }^{\mathrm{RL}} D^{\beta} f(t)$ $\in E$. In fact,

$$
{ }^{\mathrm{RL}} D^{\beta} f(t, r):=\left[{ }^{\mathrm{RL}} D^{\beta} \underline{f}(t ; r),{ }^{\mathrm{RL}} D^{\beta \bar{f}}(t ; r)\right] .
$$

Obviously, ${ }^{\mathrm{RL}} D^{\beta} f(t)=(d / d t) I^{1-\beta} f(t)$ for $t \in(0, a]$.

Definition 5 (the relation between Riemann-Liouville and Caputo's operators). Let $f(x) \in C^{(n) \mathbb{F}}[a, b] \cap L^{\mathbb{F}}[a, b]$ be a fuzzy-valued function. One defines the fuzzy fractional Caputo's derivative of order $0<\beta<1$ of $f$,

$$
\left({ }^{C} D_{a^{+}}^{\beta} f\right)(x)=\left({ }^{\mathrm{RL}} D_{a^{+}}^{\beta}\left[f(t) \ominus \sum_{k=0}^{n-1} \frac{x^{k}}{k !} f^{(k)}(a)\right]\right)(x),
$$

provided that the equation defines a fuzzy number ${ }^{C} D_{a^{+}}^{\beta} f(x)$ $\in E$ :

$$
\left({ }^{C} D_{a^{+}}^{\beta} f\right)(x ; r)=\left[\left({ }^{C} D_{a^{+}}^{\beta} \underline{f}\right)(x ; r),\left({ }^{C} D_{a^{+}}^{\beta} \bar{f}\right)(x ; r)\right],
$$

where

$$
\begin{aligned}
& \left({ }^{C} D_{a^{+}}^{\beta} \underline{f}\right)(x ; r) \\
& ={ }^{\mathrm{RL}} D_{a^{+}}^{\beta}\left[\underline{f}(t ; r)-\sum_{k=0}^{n-1} \frac{x^{k}}{k !} \underline{f}^{(k)}(a ; r)\right](x ; r) \\
& \quad=\frac{1}{\Gamma(n-\beta)} \frac{d^{n}}{d x^{n}} \int_{a}^{x} \frac{\underline{f}(t ; r)-\sum_{k=0}^{n-1}\left(t^{k} / k !\right) \underline{f}^{(k)}(a ; r)}{(x-t)^{\beta-n+1}} d t, \\
& \left({ }^{C} D_{a^{+}}^{\beta} \bar{f}\right)(x ; r) \\
& ={ }^{\mathrm{RL}} D_{a^{+}}^{\beta}\left[\bar{f}(t ; r)-\sum_{k=0}^{n-1} \frac{x^{k}}{k !} \bar{f}^{(k)}(a ; r)\right](x ; r) \\
& =\frac{1}{\Gamma(n-\beta)} \frac{d^{n}}{d x^{n}} \int_{a}^{x} \frac{\bar{f}(t, r)-\sum_{k=0}^{n-1}\left(t^{k} / k !\right) \bar{f}^{(k)}(a ; r)}{(x-t)^{\beta-n+1}} d t,
\end{aligned}
$$

for all $0 \leq r \leq 1$ and $0<\beta<1$. Obviously, ${ }^{C} D_{a^{+}}^{\beta} f(t)=$ $I^{n-\beta} D^{n} f(t)$ for $n-1<\beta<n, n \in \mathbb{N}$.

Definition 6 (see [52]). The $n \times n$ linear system is

$$
\begin{aligned}
& a_{11} x_{1}+a_{12} x_{2}+\cdots+a_{1 n} x_{n}=y_{1}, \\
& a_{11} x_{1}+a_{12} x_{2}+\cdots+a_{1 n} x_{n}=y_{1},
\end{aligned}
$$

$$
a_{n 1} x_{1}+a_{n 2} x_{2}+\cdots+a_{n n} x_{n}=y_{n},
$$

where the coefficient matrix $A=\left(a_{i j}\right), 1 \leq i, j \leq n$, is a crisp $n \times n$ matrix, and $y_{i} \in \mathbb{E}, 1 \leq i \leq n$, is called a fuzzy system of linear equations (FSLEs).

Definition 7. A fuzzy number vector $\left(x_{1}, x_{2}, \ldots, x_{n}\right)^{t}$ given by $x_{i}=\left(\underline{x}_{i}(r), \bar{x}_{i}(r)\right), 1 \leq i \leq n, 0 \leq r \leq 1$, is called a solution of the FSLE if

$$
\begin{aligned}
& \underline{\sum_{j=1}^{n} a_{i j} x_{j}}=\sum_{j=1}^{n} \frac{a_{i j} x_{j}}{y}=\underline{y}_{i}, \\
& \overline{\sum_{j=1}^{n} a_{i j} x_{j}}=\sum_{j=1}^{n} \overline{a_{i j} x_{j}}=\bar{y}_{i} .
\end{aligned}
$$

Considering the $i$ th equation of the system (13)

$$
a_{i 1}\left(\underline{x}_{1}, \bar{x}_{1}\right)+\cdots+a_{i i}\left(\underline{x}_{i}, \bar{x}_{i}\right)+\cdots+a_{i n}\left(\underline{x}_{n}, \bar{x}_{n}\right)=\left(\underline{y}_{i}, \bar{y}_{i}\right),
$$

we have

$$
\begin{array}{r}
\underline{a_{i 1} x_{1}}+\cdots+\underline{a_{i i} x_{i}}+\cdots+\underline{a_{i n} x_{n}}=\underline{y_{i}}(r), \\
\overline{a_{i 1} x_{1}}+\cdots+\overline{a_{i i} x_{i}}+\cdots+\overline{a_{i n} x_{n}}=\overline{y_{i}}(r), \\
1 \leq i \leq n, \quad 0 \leq r \leq 1 .
\end{array}
$$


From (16), we have two crisp $n \times n$ linear systems for all $i$ that can be extended to a $2 n \times 2 n$ crisp linear system as follows:

$$
\mathbf{S X}=\mathbf{Y} \Longrightarrow\left[\begin{array}{ll}
s_{1} \geq 0 & s_{2} \leq 0 \\
s_{2} \leq 0 & s_{1} \geq 0
\end{array}\right]\left[\frac{\bar{X}}{\bar{X}}\right]=\left[\frac{Y}{\bar{Y}}\right]
$$

where $s_{i j}$ are determined as follows:

$$
\begin{aligned}
& \text { if } a_{i j} \geq 0 \Longrightarrow s_{i j}=a_{i j}, s_{i+n, j+n}=a_{i j}, \\
& \text { if } a_{i j}<0 \Longrightarrow s_{i, j+n}=a_{i j}, s_{i+n, j}=a_{i j},
\end{aligned}
$$

and any $s_{i j}$ which is not determined is zero.

In this paper, the following system will be solved:

$$
\begin{gathered}
\frac{d^{\beta_{1}} \tilde{x}_{1}}{d t^{\beta_{1}}}=f_{1}\left(\tilde{x}_{1}, \tilde{x}_{2}, \ldots, \tilde{x}_{n}\right)=a_{11} \tilde{x}_{1}+a_{12} \tilde{x}_{2}+\cdots+a_{1 n} \tilde{x}_{n} \\
\frac{d^{\beta_{2}} \tilde{x}_{2}}{d t^{\beta_{2}}}=f_{2}\left(\tilde{x}_{1}, \tilde{x}_{2}, \ldots, \tilde{x}_{n}\right)=a_{21} \tilde{x}_{1}+a_{22} \tilde{x}_{2}+\cdots+a_{2 n} \tilde{x}_{n} \\
\vdots \\
\frac{d^{\beta_{n}} \tilde{x}_{n}}{d t^{\beta_{n}}}=f_{n}\left(\tilde{x}_{1}, \tilde{x}_{2}, \ldots, \tilde{x}_{n}\right)=a_{n 1} \tilde{x}_{1}+a_{n 2} \tilde{x}_{2}+\cdots+a_{n n} \tilde{x}_{n} .
\end{gathered}
$$

Thus,

$$
\frac{d^{\beta}}{d t^{\beta}} \widetilde{\mathbf{X}}(t)=A \widetilde{\mathbf{X}}(t), \quad \widetilde{\mathbf{X}}(0)=\mathbf{X}_{0} \in \mathbb{E}^{n}
$$

where $\widetilde{\mathbf{X}} \in \mathbb{E}^{n}$, the matrix $A=\left(a_{i j}\right), 1 \leq i, j \leq n, \beta=\left[\beta_{1}, \beta_{2}\right.$, $\left.\ldots, \beta_{n}\right]$ indicates the fractional orders, $d^{\beta} / d t^{\beta}=\left[d^{\beta_{1}} / d t^{\beta_{1}}\right.$, $\left.d^{\beta_{2}} / d t^{\beta_{2}}, \ldots, d^{\beta_{n}} / d t^{\beta_{n}}\right]$, and $d^{\beta_{i}} / d t^{\beta_{i}}$ is the fuzzy Caputo's fractional derivative of $\operatorname{order} \beta_{i}$, where $0<\beta_{i}<1$, for $i=1,2$, $\ldots, n$. To obtain the solution of $\left(d^{\beta} / d t^{\beta}\right) \widetilde{\mathbf{X}}(t)=A \widetilde{\mathbf{X}}(t)$, the eigenvalue-eigenvector method is used.

\section{Fuzzy Fractional Order Linear Systems}

In this section, we drive the general solution for fuzzy fractional order linear system as follows:

$$
\frac{d^{\beta}}{d t^{\beta}} \widetilde{\mathbf{X}}(t)=A \widetilde{\mathbf{X}}(t), \quad 0<t \leq a,
$$

where $\widetilde{\mathbf{X}} \in \mathbb{E}^{n}, a>0$, the coefficient matrix $A=\left(a_{i j}\right), 1 \leq$ $i, j \leq n$, is a crisp $n \times n$ matrix and $\tilde{x}_{i} \in \mathbb{E}, 1 \leq i \leq n$, and $d^{\beta} / d t^{\beta}$ is the fuzzy Caputo's fractional derivative, where $0<\beta<1$. Simply to construct the general solution of the system (21), we proceed by analogy with treatment of homogeneous integer order fuzzy linear systems with the constant coefficient where the exponential function $\operatorname{Exp}(t)$ is replaced by the Mittag-Leffler function $E_{\beta}\left(t^{\beta}\right)$. Thus, we seek solutions of the form

$$
\widetilde{\mathbf{X}}(t)=\xi E_{\beta}\left(\lambda t^{\beta}\right)
$$

where the constant $\lambda$ and the vector $\xi$ are to be determined. Substituting form (22) for $\mathbf{X}$ in the system (21) gives

$$
\xi \lambda E_{\beta}\left(\lambda t^{\beta}\right)=A \xi E_{\beta}\left(\lambda t^{\beta}\right)
$$

Upon canceling the nonzero factor $E_{\beta}\left(\lambda t^{\beta}\right)$, we obtain $A \xi=$ $\lambda \xi$ or

$$
(A-\lambda I) \xi=0
$$

where $I$ is the $n \times n$ identity matrix. Therefore, the vector $\widetilde{\mathbf{X}}$ given by (22) is a solution of the system (21) provided that $\lambda$ and the vector $\xi$ are associated eigenvectors of the matrix $A$. In the following Section, three cases for the eigenvalue of matrix $A$ are discussed.

4.1. Real and Distinct Eigenvalues. In this case, suppose that $\lambda_{i}$, for $i=1, \ldots, n$, are the real eigenvalues of matrix $A$. Therefore, the solution of (20) is as follows:

$$
\widetilde{\mathbf{X}}(t)=\sum_{i=1}^{n} \widetilde{c}_{i} v_{i}(t)
$$

where $\widetilde{c}_{i}$ are fuzzy numbers, $v_{i}(t)=E_{\beta}\left(\lambda_{i} t^{\beta}\right) \xi_{i}$ for $i=1, \ldots, n$ and $\lambda_{i}$ and $\xi_{i}$ are the real eigenvalues and eigenvector of matrix $A$, respectively.

By setting initial values $t=a$, in (25),

$$
\widetilde{\mathbf{X}}(a)=\sum_{i=1}^{n} \widetilde{c}_{i} E_{\beta}\left(\lambda_{i} t^{\beta}\right) \xi_{i}=\widetilde{\alpha}
$$

is obtained, where $\xi_{i}=\left[\xi_{i 1}, \xi_{i 2}, \ldots, \xi_{i n}\right]^{t}$ and $\widetilde{\alpha}=\left[\widetilde{\alpha}_{1}, \widetilde{\alpha}_{2}\right.$, $\left.\ldots, \widetilde{\alpha}_{n}\right]^{t}$. Thus, $\widetilde{c}_{i}, i=1, \ldots, n$. From the following, fuzzy systems are obtained:

$$
\begin{gathered}
\widetilde{c}_{1} E_{\beta} \lambda_{1} a^{\beta} \xi_{11}+\widetilde{c}_{2} E_{\beta} \lambda_{2} a^{\beta} \xi_{21}+\cdots+\widetilde{c}_{n} E_{\beta} \lambda_{n} a^{\beta} \xi_{n 1}=\widetilde{\alpha}_{1}, \\
\widetilde{c}_{1} E_{\beta} \lambda_{1} a^{\beta} \xi_{12}+\widetilde{c}_{2} E_{\beta} \lambda_{2} a^{\beta} \xi_{22}+\cdots+\widetilde{c}_{n} E_{\beta} \lambda_{n} a^{\beta} \xi_{n 2}=\widetilde{\alpha}_{2}, \\
\vdots \\
\widetilde{c}_{1} E_{\beta} \lambda_{1} a^{\beta} \xi_{1 n}+\widetilde{c}_{2} E_{\beta} \lambda_{2} a^{\beta} \xi_{2 n}+\cdots+\widetilde{c}_{n} E_{\beta} \lambda_{n} a^{\beta} \xi_{n n}=\widetilde{\alpha}_{2} .
\end{gathered}
$$


The parametric form of (27) is as follows:

$$
\begin{gathered}
\left(\underline{c}_{1}(r), \bar{c}_{1}(r)\right) E_{\beta} \lambda_{1} a^{\beta} \xi_{11}+\left(\underline{c}_{2}(r), \bar{c}_{2}(r)\right) E_{\beta} \lambda_{2} a^{\beta} \xi_{21} \\
+\cdots+\left(\underline{c}_{n}(r), \bar{c}_{n}(r)\right) E_{\beta} \lambda_{n} a^{\beta} \xi_{n 1}=\left(\underline{\alpha}_{1}(r), \bar{\alpha}_{1}(r)\right), \\
\left(\underline{c}_{1}(r), \bar{c}_{1}(r)\right) E_{\beta} \lambda_{1} a^{\beta} \xi_{12}+\left(\underline{c}_{2}(r), \bar{c}_{2}(r)\right) E_{\beta} \lambda_{2} a^{\beta} \xi_{22} \\
+\cdots+\left(\underline{c}_{n}(r), \bar{c}_{n}(r)\right) E_{\beta} \lambda_{n} a^{\beta} \xi_{n 2}=\left(\underline{\alpha}_{2}(r), \bar{\alpha}_{2}(r)\right), \\
\vdots \\
\left(\underline{c}_{1}(r), \bar{c}_{1}(r)\right) E_{\beta} \lambda_{1} a^{\beta} \xi_{1 n}+\left(\underline{c}_{2}(r), \bar{c}_{2}(r)\right) E_{\beta} \lambda_{2} a^{\beta} \xi_{2 n} \\
+\cdots+\left(\underline{c}_{n}(r), \bar{c}_{n}(r)\right) E_{\beta} \lambda_{n} a^{\beta} \xi_{n n}=\left(\underline{\alpha}_{n}(r), \bar{\alpha}_{n}(r)\right) .
\end{gathered}
$$

Now similar to (17), there is a $2 n \times 2 n$ crisp system. Therefore, $\widetilde{c}_{i}=\left(c_{i}(r), \bar{c}_{i}(r)\right), i=1,2, \ldots, n$ are obtained from $(28)$ and are set in (25). Finally the solution of (20) will be obtained from $\widetilde{\mathbf{X}}(t)=\left[\tilde{x}_{1}, \tilde{x}_{2}, \ldots, \tilde{x}_{n}\right]^{t}$.

Theorem 8. The solution of fuzzy system (20) with real eigenvalues is a fuzzy number (25).

Proof. It is shown that $\widetilde{\mathbf{X}}(t)=\sum_{i=1}^{n} \widetilde{c}_{i} E_{\beta}\left(\lambda_{i} t^{\beta}\right) \xi_{i}$ for $i=$ $1, \ldots, n$ and $0<\beta<1$ is the solution of $\left(d^{\beta} / d t^{\beta}\right) \widetilde{\mathbf{X}}=A \widetilde{\mathbf{X}}$. Let $\widetilde{C}=\left[\widetilde{c}_{1}, \widetilde{c}_{2}, \ldots, \widetilde{c}_{n}\right]$ with $\widetilde{c}_{i}$ which are the fuzzy numbers and $[\widetilde{C}]_{r}=\prod_{i=1}^{n}\left[\widetilde{c}_{i}\right]_{r}$, then

$$
\begin{aligned}
& \underline{X}(t ; r)=\min \left\{\sum_{i=1}^{n} c_{i} E_{\beta}\left(\lambda_{i} t^{\beta}\right) \xi_{i} \mid c_{i} \in[\widetilde{C}]_{r}\right\} \\
& =\sum_{i=1}^{n} c_{i} E_{\beta}\left(\lambda_{i} t^{\beta}\right) \xi_{i}=\sum_{i=1}^{n} \underline{c_{i} E_{\beta}\left(\lambda_{i} t^{\beta}\right) \xi_{i}}, \\
& \bar{X}(t ; r)=\max \left\{\sum_{i=1}^{n} c_{i} E_{\beta}\left(\lambda_{i} t^{\beta}\right) \xi_{i} \mid c_{i} \in[\widetilde{C}]_{r}\right\} \\
& =\overline{\sum_{i=1}^{n} c_{i} E_{\beta}\left(\lambda_{i} t^{\beta}\right) \xi_{i}}=\sum_{i=1}^{n} \overline{c_{i} E_{\beta}\left(\lambda_{i} t^{\beta}\right) \xi_{i}} .
\end{aligned}
$$

With differentiation of (29), we are obtained:

$$
\begin{array}{ll}
\frac{d^{\beta}}{d t^{\beta}} \underline{X}(t ; r)=\sum_{i=1}^{n} \overline{\lambda_{i} c_{i} E_{\beta}\left(\lambda_{i} t^{\beta}\right) \xi_{i}}, & 0 \leq r \leq 1, \\
\frac{d^{\beta}}{d t^{\beta}} \bar{X}(t ; r)=\sum_{i=1}^{n} \overline{\lambda_{i} c_{i} E_{\beta}\left(\lambda_{i} t^{\beta}\right) \xi_{i}}, & 0 \leq r \leq 1 .
\end{array}
$$

Since $\lambda_{i}$ is an eigenvalue and $\xi_{i}$ is its corresponding eigenvalue of matrix $A$, then $A \xi_{i}=\lambda \xi_{i}, i=1, \ldots, n$. Therefore,

$$
\begin{aligned}
\frac{d^{\beta}}{d t^{\beta}} \underline{X}(t ; r) & =\sum_{i=1}^{n} \frac{\lambda_{i} c_{i} E_{\beta}\left(\lambda_{i} t^{\beta}\right) \xi_{i}}{=}=\sum_{i=1}^{n} \underline{\lambda_{i} \xi_{i} c_{i} E_{\beta}\left(\lambda_{i} t^{\beta}\right)} \\
& =A \sum_{i=1}^{n} \frac{c_{i} E_{\beta}\left(\lambda_{i} t^{\beta}\right) \xi_{i}}{}=A \underline{X}(t ; r), \\
\frac{d^{\beta}}{d t^{\beta}} \bar{X}(t ; r) & =\sum_{i=1}^{n} \overline{\lambda_{i} c_{i} E_{\beta}\left(\lambda_{i} t^{\beta}\right) \xi_{i}}=\sum_{i=1}^{n} \overline{\lambda_{i} \xi_{i} c_{i} E_{\beta}\left(\lambda_{i} t^{\beta}\right)} \\
& =A \sum_{i=1}^{n} \overline{c_{i} E_{\beta}\left(\lambda_{i} t^{\beta}\right) \xi_{i}}=A \bar{X}(t ; r) .
\end{aligned}
$$

Such that

$$
\begin{aligned}
\left(\frac{d^{\beta}}{d t^{\beta}} \underline{X}(t ; r) ; \frac{d^{\beta}}{d t^{\beta}} \bar{X}(t ; r)\right) & =A(\underline{X}(t ; r) ; \bar{X}(t ; r)), \\
0 & <\beta<1, \quad 0 \leq r \leq 1 .
\end{aligned}
$$

This means that $\left(d^{\beta} / d t^{\beta}\right) \widetilde{\mathbf{X}}(t)=A \widetilde{\mathbf{X}}(t)$. From (7), (10), and $\left(d^{\beta} / d t^{\beta}\right) \widetilde{\mathbf{X}}(t)=\left[\left(d^{\beta_{1}} / d t^{\beta_{1}}\right) \tilde{x}_{1},\left(d^{\beta_{2}} / d t^{\beta_{2}}\right) \tilde{x}_{2}, \ldots\right.$, $\left.\left(d^{\beta_{n}} / d t^{\beta_{n}}\right) \tilde{x}_{n}\right]^{t}$, it is clear that $\widetilde{\mathbf{X}}(t)$ is a fuzzy number vector.

4.2. Complex Eigenvalues. In this case, suppose that some eigenvalues of $\lambda_{i}$, for $i=1,2, \ldots, k$, are complex numbers. Since the entries of matrix $A$ are real, therefore characteristic polynomial has real coefficients; therefore, complex roots are in conjugate pairs.

Lemma 9. Let the entries of matrix $A$ be real and $\lambda$ an eigenvalue of matrix $A$, where $\lambda=\mu+i v, \nu \neq 0$, and $\xi=$ $a+i b$ are the corresponding eigenvectors of $\lambda$, then $u_{1}(t)=$ $\left.\left.\operatorname{Re}\left[\xi E_{\beta}(\mu+i v) t^{\beta}\right)\right], u_{2}(t)=\operatorname{Im}\left[\xi E_{\beta}(\mu+i v) t^{\beta}\right)\right]$ are solutions.

Therefore, from the above-mentioned lemma, the solution of each pair of conjugate complex eigenvalues $\lambda=\mu+i v$ is as follows:

$$
\left.\left.\widetilde{w}(t)=\widetilde{c}_{1} \operatorname{Re}\left[\xi E_{\beta}(\mu+i v) t^{\beta}\right)\right]+\widetilde{c}_{2} \operatorname{Im}\left[\xi E_{\beta}(\mu+i \nu) t^{\beta}\right)\right],
$$

where $\xi$ is the corresponding eigenvector of eigenvalue $\lambda$. Hence, the solution of (33) is as follows:

$$
\widetilde{\mathbf{X}}(t)=\sum_{i=1}^{i=k / 2} \widetilde{w}_{i}(t)+i \sum_{i=k / 2+1}^{i=n} \widetilde{v}_{i}(t),
$$

where $\widetilde{w}_{i}(t)=\widetilde{c}_{i 1} \operatorname{Re}\left(\xi_{i} E_{\beta}\left(\lambda_{i} t^{\beta}\right)\right)+\widetilde{c}_{i 2} \operatorname{Im}\left(\xi_{i} E_{\beta}\left(\lambda_{i} t^{\beta}\right)\right)$ from each pair of conjugate complex eigenvalues and $v_{i}(t)=\widetilde{c}_{i} \xi_{i} E_{\beta}\left(\lambda_{i} t^{\beta}\right)$ from real eigenvalues are obtained. Then by setting initial values $t=a$ in (34) and by solving a fuzzy system similar to (28), fuzzy coefficients are obtained. By setting fuzzy coefficient in (34), $\widetilde{\mathbf{X}}(t)$ is obtained; finally the solution of (20) will be obtained from $\widetilde{\mathbf{X}}(t)=\left[x_{1}, x_{2}, \ldots, x_{n}\right]^{t}$. 
Theorem 10. The solution of fuzzy system (20) with complex eigenvalues is a fuzzy number (34).

Proof. It is shown that $\widetilde{\mathbf{X}}(t)=\sum_{i=1}^{i=k / 2} \widetilde{w}_{i}(t)+i \sum_{i=k / 2}^{i=n} \widetilde{v}_{i}(t)$ is the solution of $\left(d^{\beta} / d t^{\beta}\right) \widetilde{\mathbf{X}}=A \widetilde{\mathbf{X}}$.
Let $\widetilde{C}_{1}=\left(\widetilde{c}_{11}, \widetilde{c}_{21}, \ldots, \widetilde{c}_{k / 2,1}\right), \widetilde{C}_{2}=\left(\widetilde{c}_{12}, \widetilde{c}_{22}, \ldots, \widetilde{c}_{k / 2,2}\right)$, and $\widetilde{C}=\left(\widetilde{c}_{k / 2+1}, \widetilde{c}_{k / 2+2}, \ldots, \widetilde{c}_{n}\right)$ with $\widetilde{c}_{i 1}, \widetilde{c}_{i 2}, \widetilde{c}_{i}$ which are the fuzzy numbers and $\left[\widetilde{C}_{1}\right]_{r}=\Pi_{i=1}^{k / 2}\left[\widetilde{c}_{i 1}\right]_{r}, \quad\left[\widetilde{C}_{2}\right]_{r}=\Pi_{i=1}^{k / 2}\left[\widetilde{c}_{i 2}\right]_{r}$, and $[\widetilde{C}]_{r}=\prod_{i=k / 2+1}^{k / 2}\left[\widetilde{c}_{i}\right]_{r}$,

$$
\begin{aligned}
& \underline{X}(t ; r)=\min \left\{\sum_{i=1}^{k / 2} c_{i 1} \operatorname{Re}\left(\xi_{i} E_{\beta}\left(\lambda_{i} t^{\beta}\right)\right)+c_{i 2} \operatorname{Im}\left(\xi_{i} E_{\beta}\left(\lambda_{i} t^{\beta}\right)\right)+\sum_{i=k / 2+1}^{n} c_{i} \xi_{i} E_{\beta}\left(\lambda_{i} t^{\beta}\right) \mid c_{i 1} \in\left[\widetilde{C}_{1}\right]_{r}, c_{i 2} \in\left[\widetilde{C}_{2}\right]_{r}, c_{i} \in[\widetilde{C}]_{r}\right\} \\
& =\sum_{i=1}^{k / 2} c_{i 1} \operatorname{Re}\left(\xi_{i} E_{\beta}\left(\lambda_{i} t^{\beta}\right)\right)+c_{i 2} \operatorname{Im}\left(\xi_{i} E_{\beta}\left(\lambda_{i} t^{\beta}\right)\right)+\sum_{i=k / 2+1}^{n} c_{i} \xi_{i} E_{\beta}\left(\lambda_{i} t^{\beta}\right) \\
& =\sum_{i=1}^{k / 2} c_{i 1} \operatorname{Re}\left(\xi_{i} E_{\beta}\left(\lambda_{i} t^{\beta}\right)\right)+c_{i 2} \operatorname{Im}\left(\xi_{i} E_{\beta}\left(\lambda_{i} t^{\beta}\right)\right)+\sum_{i=k / 2+1}^{n} c_{i} \xi_{i} E_{\beta}\left(\lambda_{i} t^{\beta}\right), \\
& \bar{X}(t ; r)=\max \left\{\sum_{i=1}^{k / 2} c_{i 1} \operatorname{Re}\left(\xi_{i} E_{\beta}\left(\lambda_{i} t^{\beta}\right)\right)+c_{i 2} \operatorname{Im}\left(\xi_{i} E_{\beta}\left(\lambda_{i} t^{\beta}\right)\right)+\sum_{i=k / 2+1}^{n} c_{i} \xi_{i} E_{\beta}\left(\lambda_{i} t^{\beta}\right) \mid c_{i 1} \in\left[\widetilde{C}_{1}\right]_{r}, c_{i 2} \in\left[\widetilde{C_{2}}\right]_{r}, c_{i} \in[\widetilde{C}]_{r}\right\} \\
& =\overline{\sum_{i=1}^{k / 2} c_{i 1} \operatorname{Re}\left(\xi_{i} E_{\beta}\left(\lambda_{i} t^{\beta}\right)\right)+c_{i 2} \operatorname{Im}\left(\xi_{i} E_{\beta}\left(\lambda_{i} t^{\beta}\right)\right)+\sum_{i=k / 2+1}^{n} c_{i} \xi_{i} E_{\beta}\left(\lambda_{i} t^{\beta}\right)} \\
& =\sum_{i=1}^{k / 2} \overline{c_{i 1} \operatorname{Re}\left(\xi_{i} E_{\beta}\left(\lambda_{i} t^{\beta}\right)\right)+c_{i 2} \operatorname{Im}\left(\xi_{i} E_{\beta}\left(\lambda_{i} t^{\beta}\right)\right)}+\sum_{i=k / 2+1}^{n} \overline{c_{i} \xi_{i} E_{\beta}\left(\lambda_{i} t^{\beta}\right)}
\end{aligned}
$$

With differentiation of the above equations we obtain the following

$$
\begin{aligned}
\frac{d^{\beta}}{d t^{\beta}} & \underline{X}(t ; r) \\
= & \sum_{i=1}^{k / 2} \frac{c_{i 1} \operatorname{Re}\left(\xi_{i} \lambda_{i} E_{\beta}\left(\lambda_{i} t^{\beta}\right)\right)+c_{i 2} \operatorname{Im}\left(\xi_{i} \lambda_{i} E_{\beta}\left(\lambda_{i} t^{\beta}\right)\right)}{} \\
& +\sum_{i=k / 2+1}^{n} \frac{c_{i} \lambda_{i} \xi_{i} E_{\beta}\left(\lambda_{i} t^{\beta}\right),}{d t^{\beta}}, \quad 0 \leq r \leq 1, \\
& =\sum_{i=1}^{k / 2} \frac{c_{i 1}}{\operatorname{Re}\left(\xi_{i} \lambda_{i} E_{\beta}\left(\lambda_{i} t^{\beta}\right)\right)}+c_{i 2} \operatorname{Im}\left(\xi_{i} \lambda_{i} E_{\beta}\left(\lambda_{i} t^{\beta}\right)\right) \\
& +\sum_{i=k / 2+1}^{n} \frac{c_{i} \lambda_{i} \xi_{i} E_{\beta}\left(\lambda_{i} t^{\beta}\right)}{}, \quad 0 \leq r \leq 1 .
\end{aligned}
$$

Since $\lambda_{i}$ is an eigenvalue and $\xi_{i}$ is its corresponding eigenvalue of matrix $A$, then $A \xi_{i}=\lambda \xi_{i}, i=1, \ldots, n$. Therefore,

$$
\begin{aligned}
\frac{d^{\beta}}{d t^{\beta}} & \underline{X}(t ; r) \\
= & \sum_{i=1}^{k / 2} c_{i 1} \operatorname{Re}\left(A \xi_{i} E_{\beta}\left(\lambda_{i} t^{\beta}\right)\right)+c_{i 2} \operatorname{Im}\left(A \xi_{i} E_{\beta}\left(\lambda_{i} t^{\beta}\right)\right) \\
& +\sum_{i=k / 2+1}^{n} \frac{c_{i} A \xi_{i} E_{\beta}\left(\lambda_{i} t^{\beta}\right)}{}
\end{aligned}
$$$$
\frac{d^{\beta}}{d t^{\beta}} \bar{X}(t ; r)
$$$$
=\sum_{i=1}^{k / 2} \overline{c_{i 1} \operatorname{Re}\left(\xi_{i} E_{\beta}\left(\lambda_{i} t^{\beta}\right)\right)+c_{i 2} \operatorname{Im}\left(\xi_{i} E_{\beta}\left(\lambda_{i} t^{\beta}\right)\right)}
$$$$
+\sum_{i=k / 2+1}^{n} \overline{c_{i} \xi_{i} E_{\beta}\left(\lambda_{i} t^{\beta}\right)}
$$$$
=A\left(\sum_{i=1}^{k / 2} \overline{c_{i 1} \operatorname{Re}\left(\xi_{i} E_{\beta}\left(\lambda_{i} t^{\beta}\right)\right)+c_{i 2} \operatorname{Im}\left(\xi_{i} E_{\beta}\left(\lambda_{i} t^{\beta}\right)\right)}\right.
$$$$
\left.+\sum_{i=k / 2+1}^{n} \overline{c_{i} \xi_{i} E_{\beta}\left(\lambda_{i} t^{\beta}\right)}\right)=A \bar{X}(t ; r) .
$$

Then,

$$
\begin{aligned}
\left(\frac{d^{\beta}}{d t^{\beta}} \underline{X}(t ; r) ; \frac{d^{\beta}}{d t^{\beta}} \bar{X}(t ; r)\right) & =A(\underline{X}(t ; r) ; \bar{X}(t ; r)), \\
0 & <\beta<1, \quad 0 \leq r \leq 1 .
\end{aligned}
$$


This means that $\left(d^{\beta} / d t^{\beta}\right) \widetilde{\mathbf{X}}(t)=A \widetilde{\mathbf{X}}(t)$. From (7), (10), and $\left(d^{\beta} / d t^{\beta}\right) \widetilde{\mathbf{X}}(t)=\left[\left(d^{\beta_{1}} / d t^{\beta_{1}}\right) \widetilde{x}_{1},\left(d^{\beta_{2}} / d t^{\beta_{2}}\right) \widetilde{x}_{2}, \ldots,\left(d^{\beta_{n}} / d t^{\beta_{n}}\right)\right.$ $\left.\widetilde{x}_{n}\right]^{t}$, it is clear that $\widetilde{\mathbf{X}}(t)$ is a fuzzy number vector.

4.3. Multiple Eigenvalues. In this case, suppose that some eigenvalues of matrix $A$ are multiple. Suppose that $\lambda_{0}$ is an eigenvalue of matrix $A$ with multiplicity $k$, and the corresponding eigenvectors of eigenvalue $\lambda_{0}$ are $\xi_{1}, \xi_{2}, \ldots, \xi_{k}$, if all $\xi_{i}$ are linearly independent, then

$$
\widetilde{\mathbf{X}}(t)=\widetilde{c}_{1} \xi_{1} E_{\beta} \lambda_{0} t^{\beta}+\widetilde{c}_{2} \xi_{2} E_{\beta} \lambda_{0} t^{\beta}+\cdots+\widetilde{c}_{k} \xi_{k} E_{\beta} \lambda_{0} t^{\beta} .
$$

If $\xi_{i}$ and $m$ are linearly independent vectors, that is, $m<k$, then the following lemma is brought.

Lemma 11. Let $\lambda_{0}$ be an eigenvalue of matrix $A$ with multiple $k>1$, and let the numbers of $\xi_{i}$ which are linearly independent be less than $m_{0}$, therefore at least one non-zero vector exists such that

$$
(A-\lambda I) \xi \neq 0, \quad(A-\lambda I)^{2} \xi=0 .
$$

If $\xi$ is satisfied in (40), the solution is as follows:

$$
v^{\prime}(t)=E_{\beta}^{(1)}\left(\lambda_{0} t^{\beta}\right) t^{\beta}\left(A-\lambda_{0} I\right) \xi+\xi E_{\beta}\left(\lambda_{0} t^{\beta}\right),
$$

based on the properties of the Mittag-Leffler type functions, where

$$
E_{\beta}^{(1)}(z)=\frac{d}{d t} E_{\beta}(z) .
$$

In general, if matrix $A$ has a repeated eigenvalue $\lambda_{0}$ of multiplicity $k$ with $m$ linearly independent eigenvectors, where $m<k$, then the following

$$
\begin{aligned}
& x_{1}(t)=\xi_{1} E_{\beta}\left(\lambda_{0} t^{\beta}\right), \\
& x_{2}(t)=\xi_{1} t^{\beta} E_{\beta}^{(1)}\left(\lambda_{0} t^{\beta}\right)+\xi_{2} E_{\beta}\left(\lambda_{0} t^{\beta}\right), \\
& \vdots \\
& x_{k-m}(t)=\xi_{1} t^{\beta(k-m-1)} E_{\beta}^{(k-m-1)}\left(\lambda_{0} t^{\beta}\right) \\
& +\xi_{2} t^{\beta(k-m-2)} E_{\beta}^{(k-m-2)}\left(\lambda_{0} t^{\beta}\right) \\
& +\cdots+\xi_{k-m} E_{\beta}\left(\lambda_{0} t^{\beta}\right),
\end{aligned}
$$

are $k-m$ linearly independent solutions of the system (20).

Hence, with the above-mentioned lemma, the solution of (20) is as follows:

$$
\widetilde{\mathbf{X}}(t)=\sum_{i=1}^{i=k} \widetilde{c}_{i} v_{i}^{\prime}(t)+\sum_{i=k+1}^{i=n} \widetilde{c}_{i} v_{i}(t),
$$

where $v_{i}^{\prime}(t)=E_{\beta}^{(k)}\left(\lambda_{i} t^{\beta}\right) t^{\beta}\left(A-\lambda_{i} I\right) \xi_{i}+\xi_{i} E_{\beta}\left(\lambda_{i} t^{\beta}\right)$ for $\lambda_{i}$ which are satisfied in Lemma 11 and $v_{i}(t)=\xi_{i} E_{\beta}\left(\lambda_{i} t^{\beta}\right)$ for real eigenvalues are obtained. Then by setting initial values $t=a$, in (44) and by solving a fuzzy system similar to (28), fuzzy coefficient is obtained. By setting fuzzy coefficient in (44), $\widetilde{\mathbf{X}}(t)$ is obtained, and finally the solution of (20) will be obtained from $\widetilde{\mathbf{X}}(t)=\left[\widetilde{x}_{1}, \widetilde{x}_{2}, \ldots, \widetilde{x}_{n}\right]^{t}$.

Theorem 12. The solution of fuzzy system (20) with multiple eigenvalues is a fuzzy number (44).

Proof. It is shown that $\widetilde{\mathbf{X}}(t)=\sum_{i=1}^{i=k} \widetilde{c}_{i} v_{i}^{\prime}(t)+\sum_{i=k+1}^{i=n} \widetilde{c}_{i} v_{i}(t)$ is the solution of $\left(d^{\beta} / d t^{\beta}\right) \widetilde{\mathbf{X}}=A \widetilde{\mathbf{X}}$. Let $\widetilde{C}=\left[\widetilde{c}_{1}, \widetilde{c}_{2}, \ldots, \widetilde{c}_{n}\right]$ with $\widetilde{c}_{i}$ which are the fuzzy numbers and $[\widetilde{C}]_{r}=\prod_{i=1}^{n}\left[\widetilde{c}_{i}\right]_{r}$, then

$$
\begin{aligned}
\underline{X}(t ; r)= & \min \left\{\sum_{i=1}^{k} c_{i} E_{\beta}^{(k)}\left(\lambda_{i} t^{\beta}\right)\left(t^{\beta}\left(A-\lambda_{i} I\right) \xi_{i}\right)+\xi_{i} E_{\beta}\left(\lambda_{i} t^{\beta}\right)+\sum_{i=k+1}^{i=n} c_{i} \xi_{i} E_{\beta}\left(\lambda_{i} t^{\beta}\right) \mid c_{i} \in[\widetilde{C}]_{r}\right\} \\
= & \sum_{i=1}^{k} c_{i} E_{\beta}^{(k)}\left(\lambda_{i} t^{\beta}\right)\left(t^{\beta}\left(A-\lambda_{i} I\right) \xi_{i}\right)+\xi_{i} E_{\beta}\left(\lambda_{i} t^{\beta}\right)+\sum_{i=k+1}^{i=n} c_{i} \xi_{i} E_{\beta}\left(\lambda_{i} t^{\beta}\right) \\
= & \sum_{i=1}^{k} c_{i} E_{\beta}^{(k)}\left(\lambda_{i} t^{\beta}\right)\left(t^{\beta}\left(A-\lambda_{i} I\right) \xi_{i}\right)+\xi_{i} E_{\beta}\left(\lambda_{i} t^{\beta}\right)+\sum_{i=k+1}^{i=n} c_{i} \xi_{i} E_{\beta}\left(\lambda_{i} t^{\beta}\right), \\
\bar{X}(t ; r)= & \max \left\{\sum_{i=1}^{k} c_{i} E_{\beta}^{(k)}\left(\lambda_{i} t^{\beta}\right)\left(t^{\beta}\left(A-\lambda_{i} I\right) \xi_{i}\right)+\xi_{i} E_{\beta}\left(\lambda_{i} t^{\beta}\right)+\sum_{i=k+1}^{i=n} c_{i} \xi_{i} E_{\beta}\left(\lambda_{i} t^{\beta}\right) \mid c_{i} \in[\widetilde{C}]_{r}\right\} \\
= & \frac{\sum_{i=1}^{k} c_{i} E_{\beta}^{(k)}\left(\lambda_{i} t^{\beta}\right)\left(t^{\beta}\left(A-\lambda_{i} I\right) \xi_{i}\right)+\xi_{i} E_{\beta}\left(\lambda_{i} t^{\beta}\right)+\sum_{i=k+1}^{i=n} c_{i} \xi_{i} E_{\beta}\left(\lambda_{i} t^{\beta}\right)}{i=n} \frac{\sum_{i=k+1}}{c_{i} \xi_{i} E_{\beta}\left(\lambda_{i} t^{\beta}\right)} . \\
= & \sum_{i=1}^{k} \frac{c_{i} E_{\beta}^{(k)}\left(\lambda_{i} t^{\beta}\right)\left(t^{\beta}\left(A-\lambda_{i} I\right) \xi_{i}\right)+\xi_{i} E_{\beta}\left(\lambda_{i} t^{\beta}\right)}{i}
\end{aligned}
$$




$$
\begin{aligned}
\frac{d^{\beta}}{d t^{\beta}} \underline{X}(t ; r)= & \sum_{i=1}^{k} \frac{c_{i}\left[\lambda_{i} E_{\beta}^{(k)}\left(\lambda_{i} t^{\beta}\right)\left(t^{\beta}\left(A-\lambda_{i} I\right) \xi_{i}+E_{\beta}^{(k)}\left(\lambda_{i} t^{\beta}\right)\left(A-\lambda_{i} I\right) \xi_{i}+\xi_{i} \lambda_{i} E_{\beta}\left(\lambda_{i} t^{\beta}\right)\right]\right.}{} \\
& +\sum_{i=k+1}^{i=n} c_{i} \xi_{i} \lambda_{i} E_{\beta}\left(\lambda_{i} t^{\beta}\right), \\
\frac{d^{\beta}}{d t^{\beta}} \bar{X}(t ; r)= & \sum_{i=1}^{k} \overline{c_{i}\left[\lambda_{i} E_{\beta}^{(k)}\left(\lambda_{i} t^{\beta}\right)\left(t^{\beta}\left(A-\lambda_{i} I\right) \xi_{i}+E_{\beta}^{(k)}\left(\lambda_{i} t^{\beta}\right)\left(A-\lambda_{i} I\right) \xi_{i}+\xi_{i} \lambda_{i} E_{\beta}\left(\lambda_{i} t^{\beta}\right)\right]\right.} \\
& +\sum_{i=k+1}^{i=n} \overline{c_{i} \xi_{i} \lambda_{i} E_{\beta}\left(\lambda_{i} t^{\beta}\right)} .
\end{aligned}
$$

Since $\lambda_{i}$ is an eigenvalue and $\xi_{i}$ is its corresponding eigenvalue of matrix $A$, then $A \xi_{i}=\lambda \xi_{i}$. Therefore,

$$
\begin{aligned}
\left(\frac{d^{\beta}}{d t^{\beta}} \underline{X}(t ; r) ; \frac{d^{\beta}}{d t^{\beta}} \bar{X}(t ; r)\right) & =A(\underline{X}(t ; r) ; \bar{X}(t ; r)), \\
0 & <\beta<1, \quad 0 \leq r \leq 1 .
\end{aligned}
$$

This means that $\left(d^{\beta} / d t^{\beta}\right) \widetilde{\mathbf{X}}(t)=A \widetilde{\mathbf{X}}(t)$. From (7), (10), $\left(d^{\beta} / d t^{\beta}\right) \widetilde{\mathbf{X}}(t)=\left[\left(d^{\beta_{1}} / d t^{\beta_{1}}\right) \widetilde{x}_{1},\left(d^{\beta_{2}} / d t^{\beta_{2}}\right) \widetilde{x}_{2}, \ldots,\left(d^{\beta_{n}} / d t^{\beta_{n}}\right)\right.$ $\left.\widetilde{x}_{n}\right]^{t}$, it is clear that $\widetilde{\mathbf{X}}(t)$ is a fuzzy number vector.

\section{Examples}

Example 1. Consider that the system is with initial value

$$
\begin{gathered}
\left(\begin{array}{c}
\frac{d^{\beta} \tilde{x}}{d t^{\beta}} \\
\frac{d^{\beta} \tilde{y}}{d t^{\beta}}
\end{array}\right)=A\left(\begin{array}{c}
\tilde{x} \\
\tilde{y}
\end{array}\right), \quad A=\left(\begin{array}{ll}
2 & -1 \\
4 & -3
\end{array}\right), \\
\left(\begin{array}{l}
\tilde{x}(0) \\
\tilde{y}(0)
\end{array}\right)=\left(\begin{array}{l}
r+1 \\
3-r
\end{array}\right),
\end{gathered}
$$

where $0<\beta<1$.

The eigenvalues of the matrix $A$ are $\lambda_{1}=1$, and $\lambda_{2}=-2$ and their corresponding eigenvectors are $x_{1}=[1,1]^{t}$, and $x_{2}=[1,4]^{t}$, respectively. Therefore, the general solution of the system (48) is

$$
\widetilde{\mathbf{X}}(t)=\widetilde{c}_{1}\left(\begin{array}{l}
1 \\
1
\end{array}\right) E_{\beta}\left(t^{\beta}\right)+\widetilde{c}_{2}\left(\begin{array}{l}
1 \\
4
\end{array}\right) E_{\beta}\left(-2 t^{\beta}\right),
$$

where $\widetilde{c}_{1}$ and $\widetilde{c}_{2}$ are fuzzy coefficients. This is obtained by setting the initial values in parametric form, then the following system is obtained:

$$
\left[\begin{array}{llll}
1 & 1 & 0 & 0 \\
1 & 4 & 0 & 0 \\
0 & 0 & 1 & 1 \\
0 & 0 & 1 & 4
\end{array}\right] \cdot\left[\begin{array}{l}
\underline{c}_{1} \\
\underline{c}_{2} \\
\bar{c}_{1} \\
\bar{c}_{2}
\end{array}\right]=\left[\begin{array}{l}
r+1 \\
2+r \\
3-r \\
5-r
\end{array}\right],
$$

where $\widetilde{c}_{1}=(r+2 / 3 ; 7 / 3-r)$ and $\widetilde{c}_{2}=(1 / 3 ; 2 / 3)$ are obtained from the above system and are set in (25). Therefore, the solution of SFFDEs is as follows:

$$
\begin{aligned}
\widetilde{\mathbf{X}}(t)= & \left(\left(r+\frac{2}{3}\right) E_{\beta}\left(t^{\beta}\right)+\left(\frac{1}{3}\right) E_{\beta}\left(-2 t^{\beta}\right),\right. \\
& \left.\left(\frac{7}{3}-r\right) E_{\beta}\left(t^{\beta}\right)+4\left(\frac{2}{3}\right) E_{\beta}\left(-2 t^{\beta}\right)\right) .
\end{aligned}
$$

Example 2. Consider that the system is with initial value

$$
\left(\begin{array}{l}
\frac{d^{\beta} \tilde{x}}{d t^{\beta}} \\
\frac{d^{\beta} \tilde{y}}{d t^{\beta}} \\
\frac{d^{\beta} \widetilde{z}}{d t^{\beta}}
\end{array}\right)=A\left(\begin{array}{c}
\tilde{x} \\
\tilde{y} \\
\widetilde{z}
\end{array}\right), \quad A=\left(\begin{array}{ccc}
0 & 1 & 0 \\
0 & 0 & 1 \\
5 & -3 & -1
\end{array}\right),
$$

where $0<\beta<1$.

The eigenvalues of the matrix $A$ are $\lambda_{1}=1, \lambda_{2}=-1+2 i$, and $\lambda_{3}=-1-2 i$, and their corresponding eigenvectors are $\xi_{1}=[1,1,1]^{t}, \xi_{2}=[(-3 / 25)+(4 / 25) i,(-1 / 5)-$ $(2 / 5) i, 1]^{t}$, and $\xi_{3}=[(-3 / 25)-(4 / 25) i,(-1 / 5)+(2 / 5) i, 1]^{t}$, which are eigenvalues and eigenvectors of matrix $A$, respectively, and by setting the initial values in parametric form, the following system is obtained:

$$
\left[\begin{array}{cccccc}
1 & 0 & \frac{4}{25} & 0 & \frac{-3}{25} & 0 \\
1 & 0 & 0 & 0 & \frac{-1}{5} & \frac{-2}{5} \\
1 & 1 & 0 & 0 & 0 & 0 \\
0 & \frac{-3}{25} & 0 & 1 & 0 & \frac{4}{25} \\
0 & \frac{-1}{5} & \frac{-2}{5} & 1 & 0 & 0 \\
0 & 0 & 0 & 1 & 1 & 0
\end{array}\right] \cdot\left[\begin{array}{c}
\underline{c}_{1} \\
\underline{c}_{2} \\
\underline{c}_{3} \\
\bar{c}_{1} \\
\bar{c}_{2} \\
\bar{c}_{3}
\end{array}\right]=\left[\begin{array}{c}
0.75+0.25 r \\
1.5+0.5 r \\
3.75+0.25 r \\
1.25-0.25 r \\
2.5-0.5 r \\
4.25-0.25 r
\end{array}\right],
$$




$$
\begin{gathered}
\widetilde{c}_{1}=(1.553571428+0.071428 r, \\
1.696428572-0.0714285714 r), \\
\widetilde{c}_{2}=(2.196428572+0.1785714286 r, \\
2.553571428-0.1785714286 r), \\
\widetilde{c}_{3}=(-3.107142858+0.9821428567 r, \\
-1.142857142-0.9821428527 r),
\end{gathered}
$$

which are obtained from the above system and are set in (25). Therefore, the solution of SFFDEs is as follows:

$$
\begin{aligned}
& \underline{X}(t ; r)=(1.553571428+0.071428 r) E_{\beta}\left(t^{\beta}\right) \\
& +\frac{(2.196428572+0.1785714286 r)}{2}\left(\begin{array}{c}
\frac{-3}{25} \\
\frac{-1}{5} \\
1
\end{array}\right) \\
& \times\left(E_{\beta}\left((-1+2 i) t^{\beta}\right)+E_{\beta}\left((-1-2 i) t^{\beta}\right)\right) \\
& -\frac{(2.553571428-0.1785714286 r)}{2 i}\left(\begin{array}{c}
\frac{4}{25} \\
\frac{-2}{5} \\
0
\end{array}\right) \\
& \times\left(E_{\beta}\left((-1+2 i) t^{\beta}\right)-E_{\beta}\left((-1-2 i) t^{\beta}\right)\right) \\
& +\frac{(-3.107142858+0.9821428567 r)}{2}\left(\begin{array}{c}
\frac{4}{25} \\
\frac{-2}{5} \\
0
\end{array}\right) \\
& \times\left(E_{\beta}\left((-1+2 i) t^{\beta}\right)+E_{\beta}\left((-1-2 i) t^{\beta}\right)\right) \\
& +\frac{(-3.107142858+0.9821428567 r)}{2 i}\left(\begin{array}{c}
\frac{-3}{25} \\
\frac{-1}{5} \\
1
\end{array}\right) \\
& \times\left(E_{\beta}\left((-1+2 i) t^{\beta}\right)-E_{\beta}\left((-1-2 i) t^{\beta}\right)\right), \\
& \bar{X}(t ; r)=(1.696428572-0.0714285714 r) E_{\beta}\left(t^{\beta}\right) \\
& \begin{array}{l}
+\frac{(2.553571428-0.1785714286 r)}{2}\left(\begin{array}{c}
\frac{-3}{25} \\
\frac{-1}{5} \\
1
\end{array}\right) \\
\times\left(E_{\beta}\left((-1+2 i) t^{\beta}\right)+E_{\beta}\left((-1-2 i) t^{\beta}\right)\right)
\end{array}
\end{aligned}
$$

$$
\begin{aligned}
& -\frac{(2.196428572+0.1785714286 r)}{2 i}\left(\begin{array}{c}
\frac{4}{25} \\
\frac{-2}{5} \\
0
\end{array}\right) \\
& \times\left(E_{\beta}\left((-1+2 i) t^{\beta}\right)-E_{\beta}\left((-1-2 i) t^{\beta}\right)\right) \\
& +\frac{(-1.142857142-0.9821428527 r)}{2}\left(\begin{array}{c}
\frac{4}{25} \\
\frac{-2}{5} \\
0
\end{array}\right) \\
& \times\left(E_{\beta}\left((-1+2 i) t^{\beta}\right)+E_{\beta}\left((-1-2 i) t^{\beta}\right)\right) \\
& +\frac{(-1.142857142-0.9821428527 r)}{2 i}\left(\begin{array}{c}
\frac{-3}{25} \\
\frac{-1}{5} \\
1
\end{array}\right)
\end{aligned}
$$

Example 3. Consider the system

$$
\left(\begin{array}{c}
\frac{d^{\beta} \tilde{x}}{d t^{\beta}} \\
\frac{d^{\beta} \tilde{y}}{d t^{\beta}} \\
\frac{d^{\beta} \widetilde{z}}{d t^{\beta}}
\end{array}\right)=B\left(\begin{array}{c}
\tilde{x} \\
\tilde{y} \\
\widetilde{z}
\end{array}\right), \quad B=\left(\begin{array}{ccc}
1 & 1 & 1 \\
2 & 1 & -1 \\
0 & -1 & 1
\end{array}\right),
$$

where $0<\beta<1$.

The eigenvalues of the matrix $A$ are $\lambda_{1}=1, \lambda_{2}=$ $\lambda_{3}=2$, and their corresponding eigenvectors are $\xi_{1}=$ $[-3,4,2]^{t}, \xi_{2}=\xi_{3}=[0,1,-1]^{t}$, respectively. Therefore, the general solution of the system (55) is

$$
\begin{aligned}
\widetilde{\mathbf{X}}(t)= & \widetilde{c}_{1}\left(\begin{array}{c}
-3 \\
4 \\
2
\end{array}\right) E_{\beta}\left(-t^{\beta}\right)+\widetilde{c}_{2}\left(\begin{array}{c}
0 \\
1 \\
-1
\end{array}\right) E_{\beta}\left(2 t^{\beta}\right) \\
& +\widetilde{c}_{3}\left[\left(\begin{array}{c}
0 \\
1 \\
-1
\end{array}\right) t^{\beta} E_{\beta}^{(1)}\left(2 t^{\beta}\right)+\left(\begin{array}{l}
1 \\
0 \\
1
\end{array}\right) E_{\beta}\left(2 t^{\beta}\right)\right],
\end{aligned}
$$

where $\widetilde{c}_{1}, \widetilde{c}_{2}$, and $\widetilde{c}_{3}$ are fuzzy coefficients. In particular, if we take $\beta=1$, then the general solution (56) can be written as

$$
\begin{aligned}
\widetilde{\mathbf{X}}(t)= & \widetilde{c}_{1}\left(\begin{array}{c}
-3 \\
4 \\
2
\end{array}\right) \exp (-t)+\widetilde{c}_{2}\left(\begin{array}{c}
0 \\
1 \\
-1
\end{array}\right) \exp (2 t) \\
& +\widetilde{c}_{3}\left[\left(\begin{array}{c}
0 \\
1 \\
-1
\end{array}\right) t \exp (2 t)+\left(\begin{array}{l}
1 \\
0 \\
1
\end{array}\right) \exp (2 t)\right]
\end{aligned}
$$




\section{Conclusion}

In this paper, we investigated an analytical method (eigenvalue-eigenvector) for solving a system of fuzzy fractional differential equation under fuzzy Caputo's derivative. To this end, we exploited generalized $\mathrm{H}$-differentiability and derived the solutions based on this concept. To illustrate the effectiveness of the proposed method, several examples were solved. From Section 5, one can conclude that the solution of the system of fuzzy fractional differential is a fuzzy number.

\section{Acknowledgments}

The authors would like to express their thanks to Professor Tofigh Allahviranloo, the lead Guest Editor, for his consideration about this paper and the anonymous reviewers for their helpful suggestions and comments.

\section{References}

[1] A. Arara, M. Benchohra, N. Hamidi, and J. J. Nieto, "Fractional order differential equations on an unbounded domain," Nonlinear Analysis: Theory, Methods \& Applications, vol. 72, no. 2, pp. 580-586, 2010.

[2] I. Yu. Babenko, Heat and Mass Transfer, Chemia, Leningrad, Russia, 1986.

[3] R. L. Bagley, "On the fractional order initial value problem and its engineering applications," in Fractional Calculus and Its Applications, K. Nishimoto, Ed., pp. 12-20, College of Engineering, Nihon University, Tokyo, Japan, 1990.

[4] H. Beyer and S. Kempfle, "Definition of physically consistent damping laws with fractional derivatives," Zeitschrift für Angewandte Mathematik und Mechanik, vol. 75, no. 8, pp. 623-635, 1995.

[5] K. Diethelm and N. J. Ford, "Analysis of fractional differential equations," Journal of Mathematical Analysis and Applications, vol. 265 , no. 2, pp. 229-248, 2002.

[6] J. He, "Nonlinear oscillation with fractional derivative and its applications," in Proceedings of the International Conference on Vibrating Engineering, pp. 288-291, Dalian, China, 1998.

[7] R. L. Bagley and P. J. Torvik, "On the appearance of the fractional derivative in the behaviour of real materials," ASME Journal of Applied Mechanics, vol. 51, no. 2, pp. 294-308, 1984.

[8] J. A. Tenreiro Machado, "Fractional derivatives: probability interpretation and frequency response of rational approximations," Communications in Nonlinear Science and Numerical Simulation, vol. 14, no. 9-10, pp. 3492-3497, 2009.

[9] T. S. Chow, "Fractional dynamics of interfaces between softnanoparticles and rough substrates," Physics Letters A, vol. 342, no. 1-2, pp. 148-155, 2005.

[10] S. Abbasbandy and A. Shirzadi, "Homotopy analysis method for multiple solutions of the fractional Sturm-Liouville problems," Numerical Algorithms, vol. 54, no. 4, pp. 521-532, 2010.

[11] B. Ahmad and J. J. Nieto, "Existence results for a coupled system of nonlinear fractional differential equations with threepoint boundary conditions," Computers \& Mathematics with Applications, vol. 58, no. 9, pp. 1838-1843, 2009.

[12] D. Băleanu, O. G. Mustafa, and R. P. Agarwal, "On the solution set for a class of sequential fractional differential equations," Journal of Physics A, vol. 43, no. 38, Article ID 385209, 2010.
[13] J. Duan, J. An, and M. Xu, "Solution of system of fractional differential equations by Adomian decomposition method," Applied Mathematics B, vol. 22, no. 1, pp. 7-12, 2007.

[14] V. Lakshmikantham, S. Leela, and J. Vasundhara Devi, Theory of Fractional Dynamic Systems, Cambridge Scientific, Cambridge, UK, 2009.

[15] V. Lakshmikantham and R. N. Mohapatra, Theory of Fuzzy Differential Equations and Inclusions, vol. 6, Taylor \& Francis, London, UK, 2003.

[16] V. Lakshmikantham and A. S. Vatsala, "Basic theory of fractional differential equations," Nonlinear Analysis: Theory, Methods \& Applications, vol. 69, no. 8, pp. 2677-2682, 2008.

[17] Z. M. Odibat, "Analytic study on linear systems of fractional differential equations," Computers \& Mathematics with Applications, vol. 59, no. 3, pp. 1171-1183, 2010.

[18] K. B. Oldham and J. Spanier, The Fractional Calculus, Academic Press, New York, NY, USA, 1974.

[19] A. A. Kilbas, H. M. Srivastava, and J. J. Trujillo, Theory and Applications of Fractional Differential Equations, vol. 204 of North-Holland Mathematics Studies, Elsevier Science, Amsterdam, The Netherlands, 2006.

[20] I. Podlubny, Fractional Differential Equations, vol. 198 of Mathematics in Science and Engineering, Academic Press, San Diego, Calif, USA, 1999.

[21] A. Arikoglu and I. Ozkol, "Solution of fractional differential equations by using differential transform method," Chaos, Solitons and Fractals, vol. 34, no. 5, pp. 1473-1481, 2007.

[22] M. P. Tripathi, V. K. Baranwal, K. Pandey Ram, and P. Singh $\mathrm{Om}$, "A new numerical algorithm to solve fractional differential equations based on operational matrix of generalized hat functions," Communications in Nonlinear Science and Numerical Simulation, vol. 18, pp. 1327-1340, 2013.

[23] A. H. Bhrawy and A. S. Alofi, "The operational matrix of fractional integration for shifted Chebyshev polynomials," Applied Mathematics Letters, vol. 26, no. 1, pp. 25-31, 2013.

[24] M. M. Meerschaert and C. Tadjeran, "Finite difference approximations for two-sided space-fractional partial differential equations," Applied Numerical Mathematics, vol. 56, no. 1, pp. 80-90, 2006.

[25] Y. Chen, M. Yi, and C. Yu, "Error analysis for numerical solution of fractional differential equation by Haar wavelets method," in Journal of Computational Science, vol. 3, pp. 367-373, 2012.

[26] T. Allahviranloo and M. B. Ahmadi, "Fuzzy Laplace transforms," Soft Computing, vol. 14, no. 3, pp. 235-243, 2010.

[27] T. Allahviranloo, S. Abbasbandy, S. Salahshour, and A. Hakimzadeh, "A new method for solving fuzzy linear differential equations," Computing, vol. 92, no. 2, pp. 181-197, 2011.

[28] T. Allahviranloo and S. Salahshour, "A new approach for solving first order fuzzy differential equations," Communications in Computer and Information Science, vol. 81, part 5,7, pp. 522-531, 2010.

[29] C. V. Negoiţă and D. A. Ralescu, Applications of Fuzzy Sets to Systems Analysis, Birkhäuser, Basel, Switzerland, 1975.

[30] J. J. Nieto, R. Rodríguez-López, and D. N. Georgiou, "Fuzzy differential systems under generalized metric spaces approach," Dynamic Systems and Applications, vol. 17, no. 1, pp. 1-24, 2008.

[31] I. Perfilieva, "Fuzzy transforms: theory and applications," Fuzzy Sets and Systems, vol. 157, no. 8, pp. 993-1023, 2006.

[32] I. Perfilieva, H. De Meyer, B. De Baets, and D. Plšková, “Cauchy problem with fuzzy initial condition and its approximate 
solution with the help of fuzzy transform," in Proceedings of the World Congress on Computational Intelligence (WCCI '08), pp. 2285-2290, IEEE Computational Intelligence Society, Hong Kong, June 2008.

[33] M. L. Puri and D. A. Ralescu, "Fuzzy random variables," Journal of Mathematical Analysis and Applications, vol. 114, no. 2, pp. 409-422, 1986.

[34] R. P. Agarwal, V. Lakshmikantham, and J. J. Nieto, "On the concept of solution for fractional differential equations with uncertainty," Nonlinear Analysis: Theory, Methods \& Applications, vol. 72, no. 6, pp. 2859-2862, 2010.

[35] S. Arshad and V. Lupulescu, "On the fractional differential equations with uncertainty," Nonlinear Analysis: Theory, Methods \& Applications, vol. 74, no. 11, pp. 3685-3693, 2011.

[36] H. Sun, X. Song, and Y. Chen, "A class of fractional dynamic systems with fuzzy order," in Proceedings of the 8th World Congress on Intelligent Control and Automation (WCICA '10), pp. 197-201, July 2010.

[37] T. Allahviranloo, S. Salahshour, and S. Abbasbandy, "Explicit solutions of fractional differential equations with uncertainty," Soft Computing, vol. 16, pp. 297-302, 2012.

[38] S. Salahshour, T. Allahviranloo, S. Abbasbandy, and D. Baleanu, "Existence and uniqueness results for fractional differential equations with uncertainty," Advances in Difference Equations, vol. 2012, article 112, 2012.

[39] A. Ahmadian, M. Suleiman, S. Salahshour, and D. Baleanu, "A Jacobi operational matrix for solving fuzzy linear fractional differential equation," Advances in Difference Equations, vol. 2013, article 104, 2013.

[40] M. Mazandarani and A. V. Kamyad, "Modified fractional Euler method for solving fuzzy fractional initial value problem," Communications in Nonlinear Science and Numerical Simulation, vol. 18, no. 1, pp. 12-21, 2013.

[41] S. Salahshour, T. Allahviranloo, and S. Abbasbandy, "Solving fuzzy fractional differential equations by fuzzy Laplace transforms," Communications in Nonlinear Science and Numerical Simulation, vol. 17, no. 3, pp. 1372-1381, 2012.

[42] T. G. Bhaskar, V. Lakshmikantham, and V. Devi, "Revisiting fuzzy differential equations," Nonlinear Analysis: Theory, Methods \& Applications, vol. 58, no. 3-4, pp. 351-358, 2004.

[43] B. Bede and S. G. Gal, "Generalizations of the differentiability of fuzzy-number-valued functions with applications to fuzzy differential equations," Fuzzy Sets and Systems, vol. 151, no. 3, pp. 581-599, 2005.

[44] B. Bede, I. J. Rudas, and A. L. Bencsik, "First order linear fuzzy differential equations under generalized differentiability," Information Sciences, vol. 177, no. 7, pp. 1648-1662, 2007.

[45] L. Stefanini and B. Bede, "Generalized Hukuhara differentiability of interval-valued functions and interval differential equations," Nonlinear Analysis: Theory, Methods \& Applications, vol. 71, no. 3-4, pp. 1311-1328, 2009.

[46] L. Stefanini, "A generalization of Hukuhara difference and division for interval and fuzzy arithmetic," Fuzzy Sets and Systems, vol. 161, no. 11, pp. 1564-1584, 2010.

[47] H.-C. Wu, "The improper fuzzy Riemann integral and its numerical integration," Information Sciences, vol. 111, no. 1-4, pp. 109-137, 1998.

[48] J. Xu, Z. Liao, and Z. Hu, "A class of linear differential dynamical systems with fuzzy initial condition," Fuzzy Sets and Systems, vol. 158, no. 21, pp. 2339-2358, 2007.
[49] M. Friedman, M. Ma, and A. Kandel, "Numerical solutions of fuzzy differential and integral equations," Fuzzy Sets and Systems, vol. 106, no. 1, pp. 35-48, 1999.

[50] M. Ma, M. Friedman, and A. Kandel, "Numerical solutions of fuzzy differential equations," Fuzzy Sets and Systems, vol. 105, no. 1, pp. 133-138, 1999.

[51] H.-J. Zimmermann, Fuzzy Set Theory and Its Applications, Kluwer Academic Publishers, Dordrecht, The Netherlands, 4th edition, 2001.

[52] T. Allahviranloo and M. Afshar Kermani, "Solution of a fuzzy system of linear equation," Applied Mathematics and Computation, vol. 175, no. 1, pp. 519-531, 2006. 


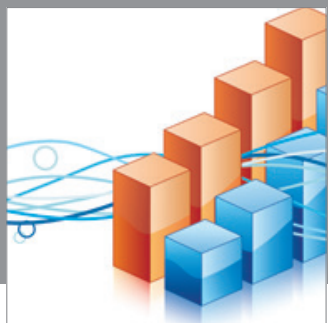

Advances in

Operations Research

mansans

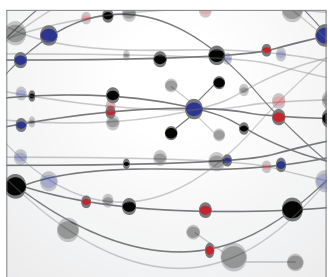

The Scientific World Journal
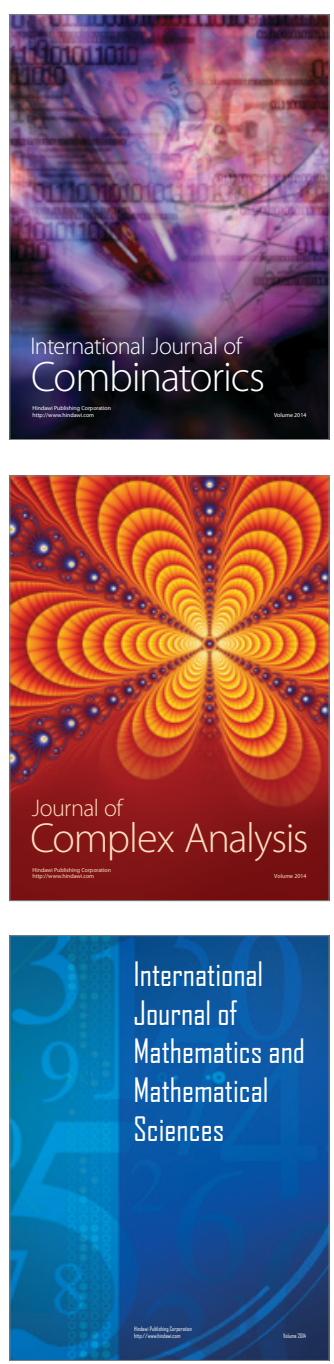
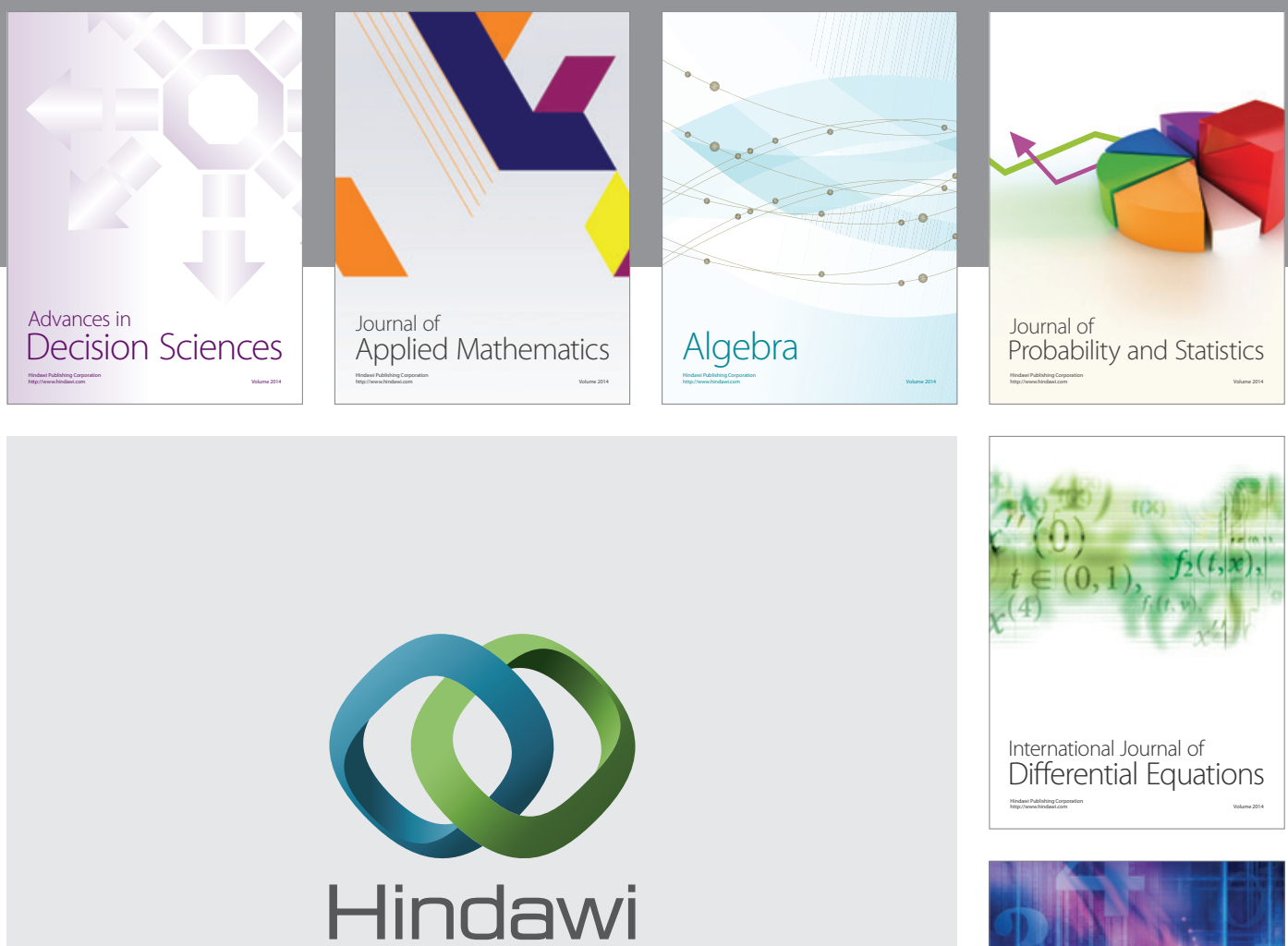

Submit your manuscripts at http://www.hindawi.com
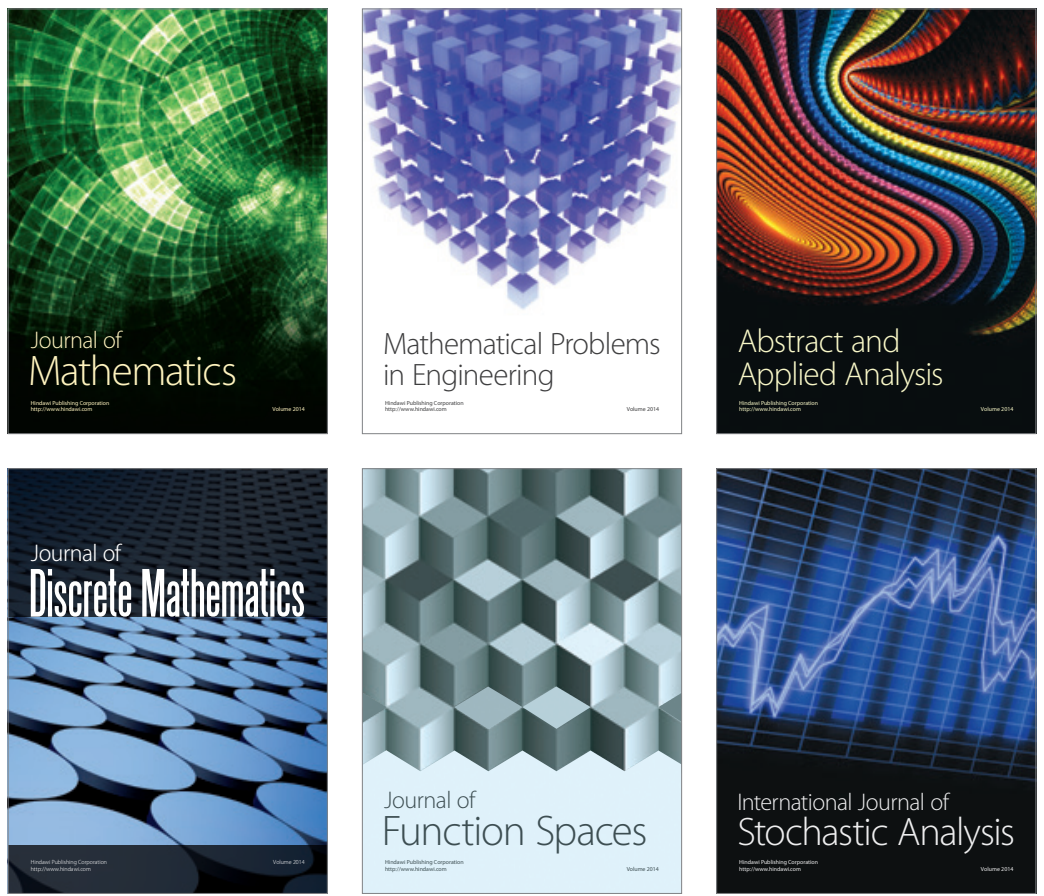

Journal of

Function Spaces

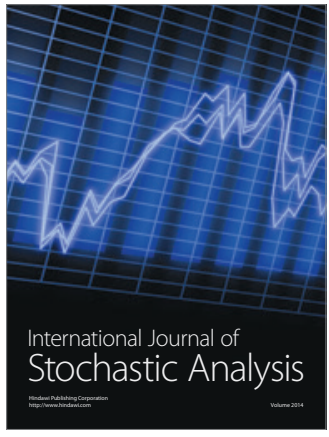

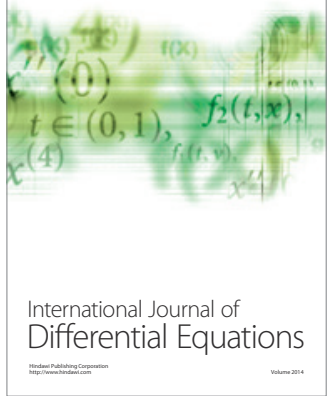
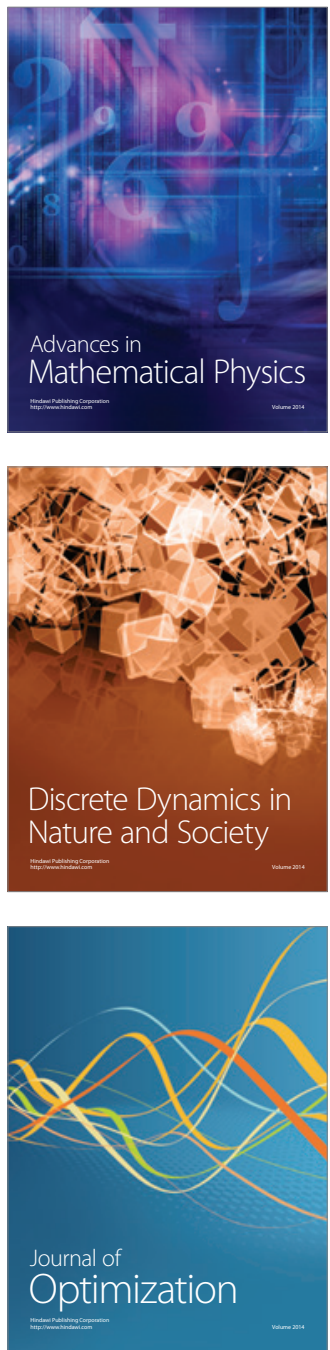